\title{
Research Paper \\ The Relationship of Substance Abuse With Depression and Stress in Diabetic Adolescents Mediated by Conflict Resolution and Problem Solving Skills
}

\author{
Ashraf Allahyar ${ }^{1}\left(\mathbb{0}\right.$, *Ali Zeinali $^{1}$ (1)
}

1. Department of Psychology, Faculty of Paramedicine,Urmia Branch, Islamic Azad University, Urmia, Iran.

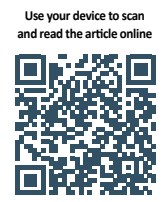

Citation: Allahyar A \& Zeinali A. [The Relationship of Substance Abuse With Depression and Stress in Diabetic Adolescents Mediated by Conflict Resolution and Problem Solving Skills (Persian)]. Journal of Arak University of Medical Sciences (JAMS). 2020; 23(2):126-137. https://doi.org/10.32598/JAMS.23.2.4087.7

doit https://doi.org/10.32598/JAMS.23.2.4087.7

Key words:

Substance abuse, Diabetes, Depression, Stress, Conflict resolution, Problem solving

\section{AB STRACT}

Article Info:

Received: 01 Nov 2019

Accepted: 15 Feb 2020

Available Online: 01 Jun 2020

Background and Aim Diabetes is one of the most common chronic diseases in children and adolescents. Due to its specific problems, it can sometimes lead to substance abuse in adolescents through reduced health and increased depression and stress. The present study aimed to investigate the relationship of substance abuse with depression and stress in diabetic adolescents mediated by conflict resolution and problem solving skills.

Methods \& Materials This is a descriptive/correlational study. The study population consisted of all diabetic adolescent members of Urmia Diabetes Association and those referred to Imam Khomeini Hospital and diabetes specialists in Urmia city in Spring 2019. Of these, 206 were selected using a purposive sampling method. data collection tools were Substance Abuse Subtle Screening Inventory - Adolescents Version (Miller \& Lazowski, 2001), Beck's Depression Inventory (Beck et al, 1988), The Perceived Stress Scale (Cohen et al, 1983), Problem Solving Inventory (Heppner \& Petersen, 1982) and Conflict Resolution Style Inventory (Weeks, 1994). Collected data were analyzed by using Pearson correlation test and structural equation modeling in SPSS and LISREL applications.

Ethical Considerations All ethical principles were considered in this study.

Results Depression and stress directly had a negative and significant effect on conflict resolution and problem solving skills, but had no significant effect on substance abuse. Conflict resolution and problem solving skills directly had a negative and significant effect on substance abuse. Moreover, depression and stress indirectly had a significant effect on substance abuse through medication by conflict resolution and problem solving skills $(\mathrm{P}<0.05)$.

Conclusion Depression and stress can lead to increased substance abuse in diabetic adolescents by decreasing conflict resolution and problem solving skills. Therefore, in order to reduce substance abuse in these patients, it is recommended to hold training workshops to reduce their depression and stress and improve conflict resolution and problem solving skills.

\section{* Corresponding Author:}

Ali Zeinali, PhD.

Address: Department of Psychology, Faculty of Paramedicine,Urmia Branch, Islamic Azad University, Urmia, Iran.

Tel: +98 (914) 3409171

E-mail: a.zeinali@iaurmia.ac.ir 


\section{Extended Abstract}

\section{Introduction}

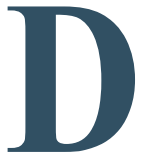

iabetes is one of the most common chronic diseases in childhood and adolescence, threatening the lives and health of patients [2] and increasing the risk of substance abuse [3]. Substance abuse is a multidimensional biological, psychological, social, and spiritual phenomenon in which several internal and external factors are involved [7]. Psychological factors associated with substance abuse include depression and stress [8]. Research has shown an association between substance abuse and depression and stress [15-18]. Conflict resolution and problem solving methods play an effective role in solving problems [22]. Different studies have indicated the relationship of stress and depression with conflict resolution and problem solving skills [27-30] and the relationship of conflict resolution and problem solving skills with substance abuse [32, 33]. This study aimed to examine the effect of stress and depression on substance abuse of adolescents with diabetes through mediation by conflict resolution and problem solving skills.

\section{Materials and Methods}

This is a descriptive/correlational study. The study population consists of all adolescents with diabetes who were are members of the Diabetes Association in Urmia city and those referred to Imam Khomeini Hospital in this city in 2019. Of these, 206 patients were determined using Structural Equation Modeling (SEM) and then selected using a purposive sampling method after obtaining informed consent from them. Inclusion criteria for them were: having diabetes, reading and writing literacy, physical health, and not experiencing stressful events such as death and divorce in the past three months. On the other hand, exclusion criteria were: having psychiatric disorders and not using the related medications, unwillingness to complete questionnaire or returning them incomplete. Data collection tools were: Substance Abuse Subtle Screening Inventory adolescent version, Beck's Depression Inventory, The Perceived Stress Scale, Problem Solving Inventory, and Conflict Resolution Style Inventory. Collected data were analyzed in SPSS V. 21 and LISREL applications by using descriptive (frequency, mean, standard deviation) and inferential (Pearson correlation test, and SEM) statistics at the significance level of $\mathrm{P}<0.05$.

\section{Results}

Of 206 participants, 110 were boys (53.40\%) and 96 girls $(46.60 \%)$. The age range of 44 subjects was $11-13$ years
(21.36\%); for 69 , 16-16 years $(33.49 \%)$, and for $93,17-19$ years $(45.15 \%)$. The relationship of substance abuse with depression and stress was positive and significant, and its relationship with conflict resolution and problem solving skills was negative and significant $(\mathrm{P}<0.01)$. All the fit indices indicated the good fit of the substance abuse reduction model in diabetic adolescents. Depression and stress variables were able to predict $13 \%$ of changes in problem-solving skills and $9 \%$ of changes in conflict resolution skills. The four variables were able to predict $46 \%$ of changes in substance abuse.

The results of SEM analysis showed that the direct effect of depression $(\beta=0.02)$ and stress $(\beta=0.04)$ on substance abuse was not significant $(\mathrm{P}>0.05)$; however, the direct effect of depression $(\beta=-0.23)$ and stress $(\beta=-0.24)$ on problem solving skills, and the direct effect of depression $(\beta=-0.16)$ and stress ( $\beta=-0.23)$ on conflict resolution skills, and the direct effect of problem solving $(\beta=-0.52)$ and conflict resolution $(\beta=-0.19)$ on substance abuse were significant. Moreover, the indirect effect of depression through problem solving $(\beta=0.13)$ and through conflict resolution $(\beta=0.04)$ on substance abuse, and the indirect effect of stress through problem solving $(\beta=0.13)$ and through conflict resolution $(\beta=0.06)$ on substance abuse were significant $(\mathrm{P}<0.05)$ (Tables $1 \& 2)$.

\section{Discussion}

The findings showed that the direct effect of depression and stress on problem-solving and conflict resolution skills in diabetic adolescents was negative and significant. This is consistent with the results of Heydari, Bayani et al., Sorati, and Ozkan [27-30]. People with depression and stress are prone to problems and lack of appropriate solutions for them due to emotional, behavioral, cognitive, and emotional problems.

The direct effect of depression and stress on substance abuse in diabetic adolescents was not significant. This was against the results of Mohammad Nia and Mashhadi, Akbari and Amoupour, Olafsdottir et al., and Esmaeelzadeh et al. [15-18]. This discrepancy may be due to the difference in study population. All of these studies were performed on school students, college students, and people without the disease, while the present study was performed on diabetic adolescents. These groups appear to have less tendency towards substance use than patients who have a problem called diabetes.

The direct effect of conflict resolution and problem-solving skills on substance abuse of study samples was negative and significant. This is consistent with the findings of Jaffee and D'Zurilla [32], and Nasrazadani et al. [33]. People who have good conflict resolution and problem-solving skills are less likely to have stress than those no such skills. These skills 
Table 1. Mean \pm SD of study variables and their correlation coefficients

\begin{tabular}{|c|c|c|c|c|c|c|}
\hline Variables & Mean $\pm S D$ & Depression & Stress & Problem Solving & Conflict Resolution & Substance Abuse \\
\hline Depression & $24.00 \pm 00.78$ & 1 & & & & \\
\hline Stress & $17.8 \pm 41.48$ & $* 0.16$ & 1 & & & \\
\hline Problem solving & $135.24 \pm 42.69$ & $* *-0.21$ & $* *-0.24$ & 1 & & \\
\hline Conflict resolution & $17.23 \pm 65.17$ & $* *-0.21$ & $* *-0.34$ & $* * 0.22$ & 1 & \\
\hline Substance abuse & $19.9 \pm 95.12$ & $* * 0.21$ & $* * 0.22$ & $* * 0.52$ & $* * 0.28$ & 1 \\
\hline
\end{tabular}

Table 2. Results of study hypotheses

\begin{tabular}{|c|c|c|c|c|}
\hline Hypothesis & $\begin{array}{l}\text { Path Coef- } \\
\text { ficient }\end{array}$ & $\begin{array}{l}\text { Standard Error of } \\
\text { Estimate }\end{array}$ & $\mathbf{t}$ & Sig. \\
\hline Depression - problem solving & -0.23 & 0.08 & -3.21 & 0.001 \\
\hline Stress - problem solving & -0.24 & 0.09 & -3.41 & 0.001 \\
\hline Depression - conflict resolution & -0.16 & 0.04 & -2.27 & 0.02 \\
\hline Stress - conflict resolution & -0.23 & 0.07 & -3.35 & 0.001 \\
\hline Depression - substance abuse & 0.02 & 0.02 & 0.34 & 0.73 \\
\hline Stress - substance abuse & 0.04 & 0.05 & 0.46 & 0.64 \\
\hline Problem solving - substance abuse & -0.52 & 0.04 & -6.88 & 0.001 \\
\hline Conflict resolution - substance abuse & -0.19 & 0.04 & -2.96 & 0.003 \\
\hline Depression - problem solving - substance abuse & 0.13 & 0.07 & 2.34 & 0.01 \\
\hline Depression - conflict resolution - substance abuse & 0.04 & 0.02 & 2.26 & 0.02 \\
\hline Stress - problem solving - substance abuse & 0.13 & 0.06 & 2.14 & 0.03 \\
\hline Stress - conflict resolution - substance abuse & 0.06 & 0.03 & 3.01 & 0.003 \\
\hline
\end{tabular}

refer to a cognitive-behavioral process that provides a variety of new and appropriate answers to deal with problematic situations and provides the basis for increasing the possibility of choosing the best and most effective response. Overall, it was concluded that depression and stress increase substance abuse in diabetic adolescents by reducing their conflict resolution and problem-solving skills.

\section{Ethical Considerations}

\section{Compliance with ethical guidelines}

All ethical principles were considered in this study including obtaining informed consent from participants, confidentiality of their information, and explaining study process to them. Also this study ethically approved in Faculty of Paramedicine,Urmia Branch, Islamic Azad University (Code: IR.IAU.URMIA.REC.1397.022)

\section{Funding}

The present paper was extracted from the MSc thesis of the first author, Department of Psychology, Urmia Branch, Islamic Azad University.

\section{Authors' contributions}

All authors met the writing standards based on the recommendations of the International Committee of Medical Journal Editors (ICMJE).

\section{Conflicts of interest}

The authors declare no conflict of interest.

\section{Acknowledgements}

The authors would like to thank the Deputy for Research of Islamic Azad University of Urmia branch, Urmia Diabetes Association, Imam Khomeini Hospital, diabetes specialists. 


\section{مدل كاهش سوءمصرف مواد در نوجوانان ديابتى: نقش افسردتى و استرس با ميانجيكَى

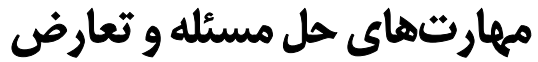

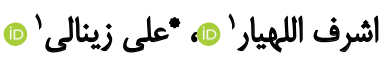

ا. كروه روانشناسى، دانشكده هيرايزشكى، واحد اروميه، دانشكاه آزاد اسلامى، اروميه، ايران.

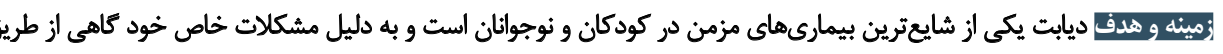

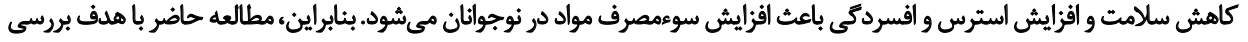

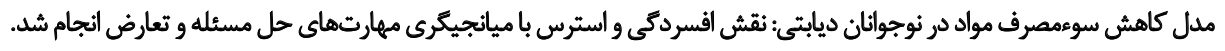

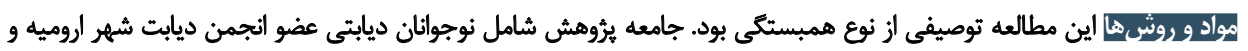

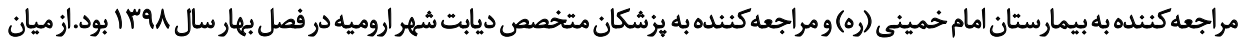

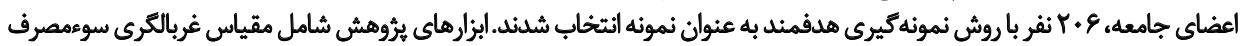

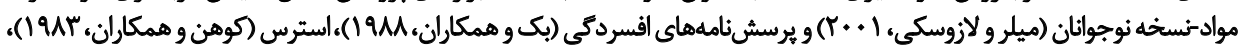

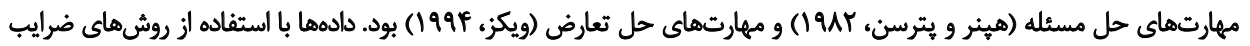

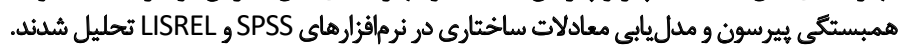

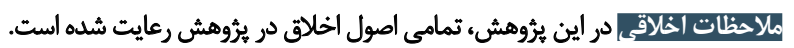

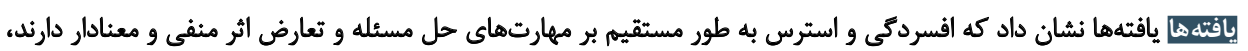

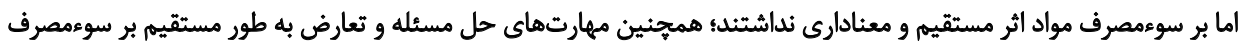

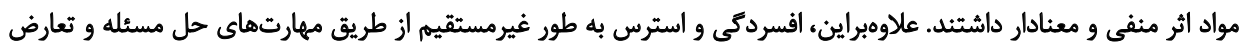

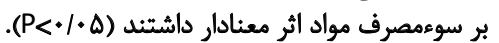

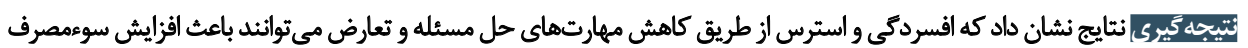

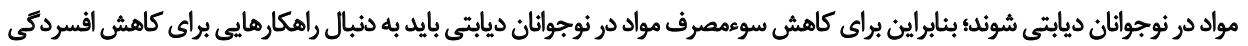

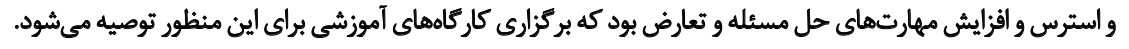

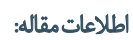

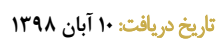

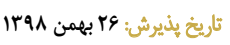

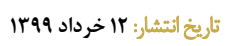

كليدوازوها: سوءمصرف مواده ديابث،

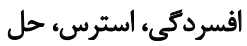
مسئله، حل تعارض الفترض حل

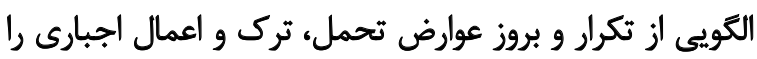

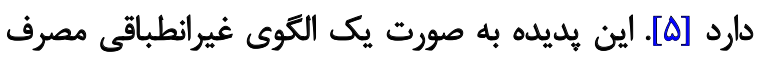

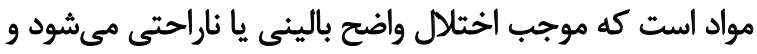

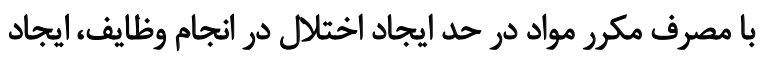

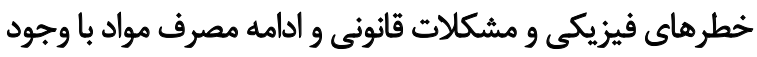

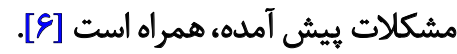

سوءمصرف مواد يك يديده جندبعدى زيستى، روانى، اجتماعى إنى

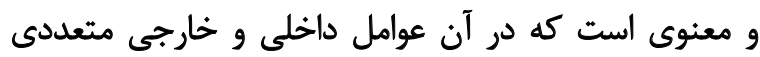

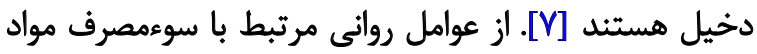

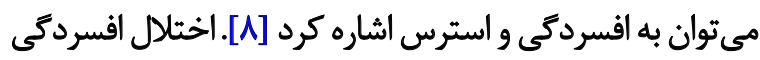

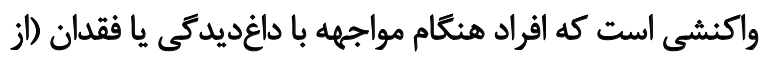

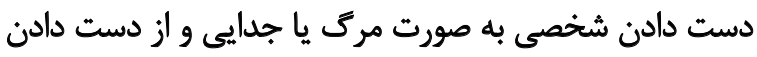

ديابت به كروهى از بيمارىهاى متابوليك كفته ميشود كه

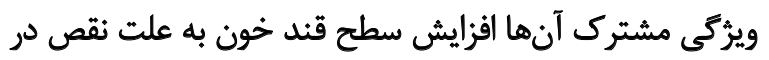

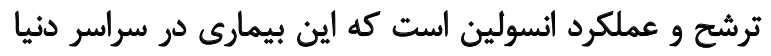

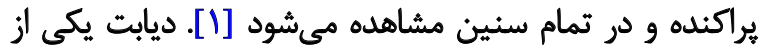

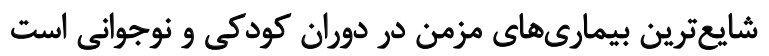

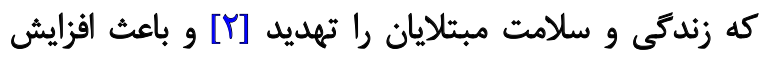

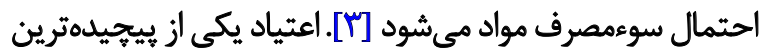

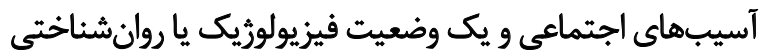

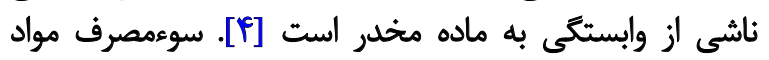
مجموعهاى از علائم شناختى، رفتارى و وروانشئ اسناختى است كه

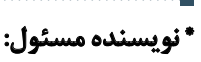

$$
\begin{aligned}
& \text { على زينالي } \\
& \text { نشانى: اروميه، دانشكاه آزاد اسلامى واحد اروميه، كروه روانشئاسى. } \\
& \text { تلفن: } \\
& \text { a.zeinali@iaurmia.ac.ir : بـت الكترونيكى }
\end{aligned}
$$


حاكى از ارتباط استرس و افسردگى با مهارتهاى حل مسئله و

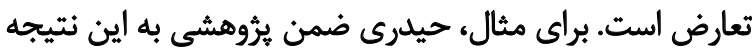

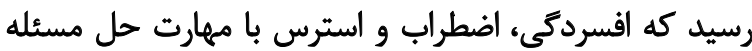

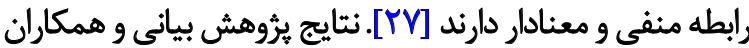

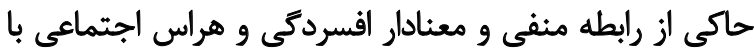

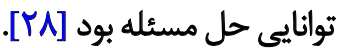

در يروهشى ديكر، صورتى كَّزارش كرد كه بين شيوههاى حل

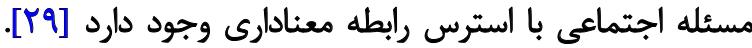

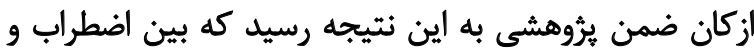

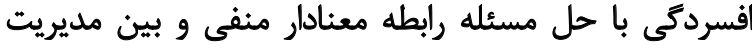

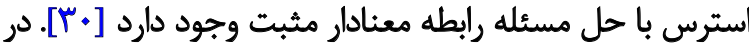

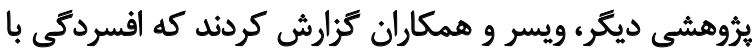

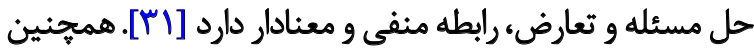
نتايج يروهش ها حاكى از ارتباط مهارتهاي حل مل مسئله و تعارض

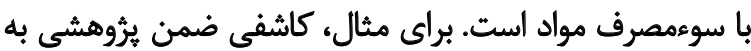

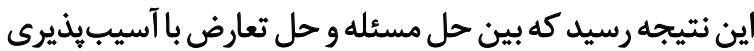

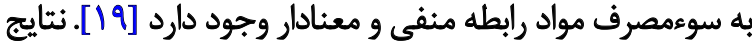

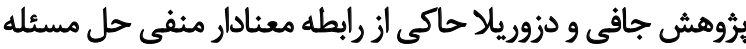

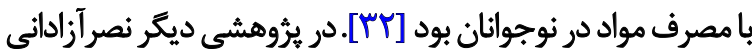

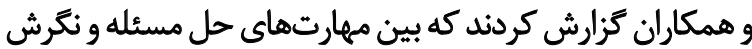

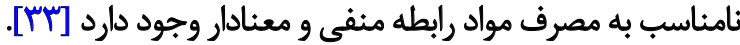
ازيكسو، نوجوانان ديابتى با مشكلات روانشناختى فراوانى

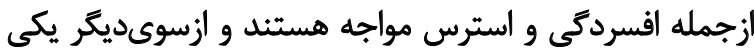

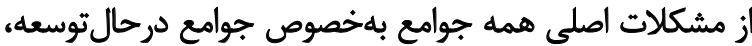

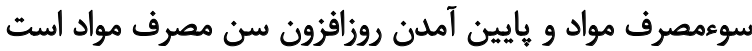

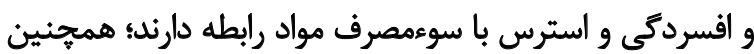

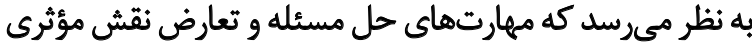

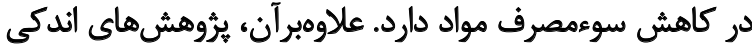

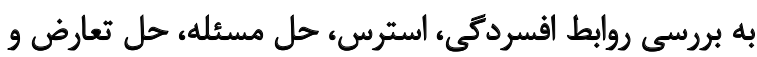

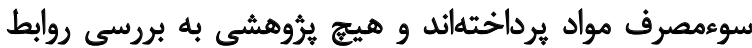

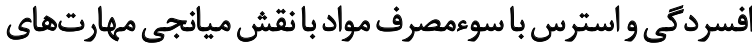

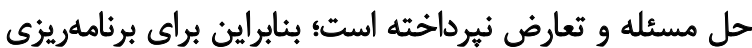

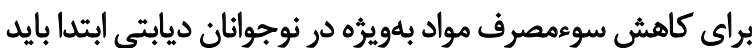

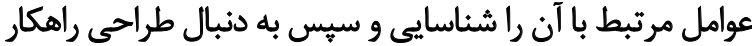

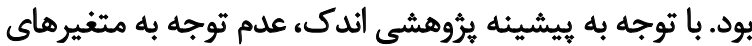

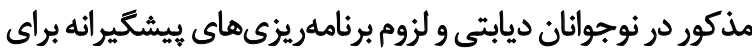

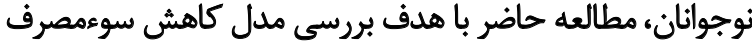

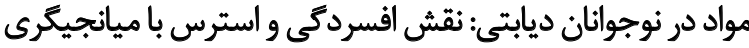
مهارتهاى حل مسئله و تعارض انجام شد.

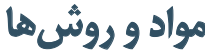

اين مطالعه توصيفى از نوع همبستَى بود. جامعه يُروهش شامل

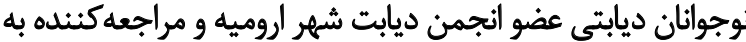

اموال) احساس مى كنند [9]. اين اختلال، اختلالى روانيزشكى دوري

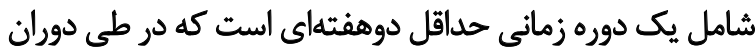

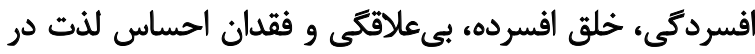

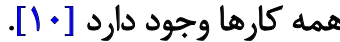

افسردئى با فقدان احساس لذت، دورى تزيدن از دوستان يا

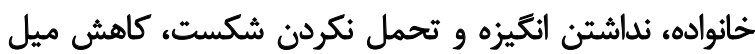

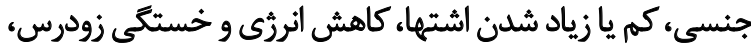

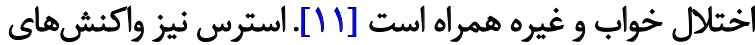

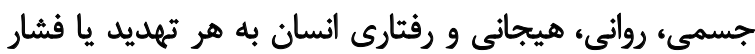

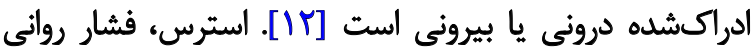

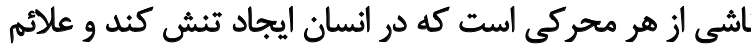

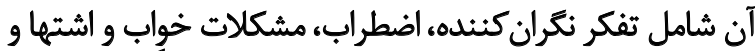

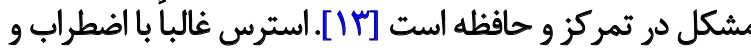

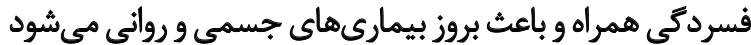

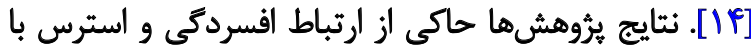

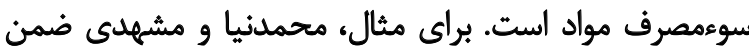

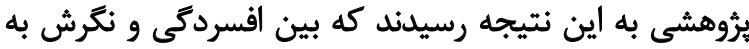

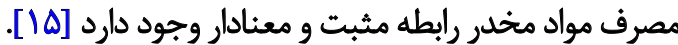
نتايج يثوهش اكبرى و عمويور حاكى از رابطه معنادار مثبت

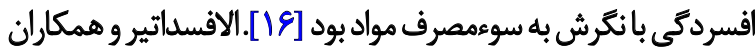

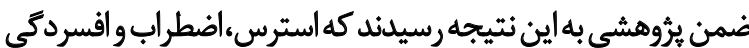

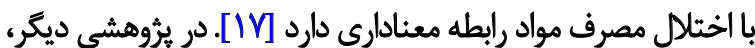

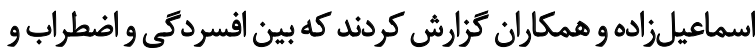

مصرف مواد رابطه منفى و معنادار وجود دارد [1/11]

مهارتهاى حل مسئله و تعارض با سوءمصرف مواد مرتبط

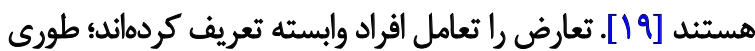

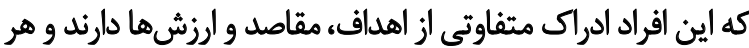

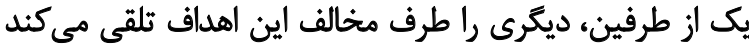

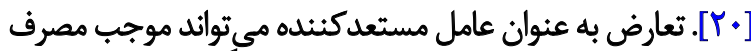

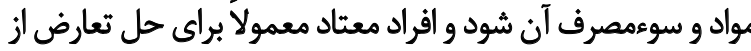

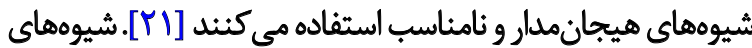

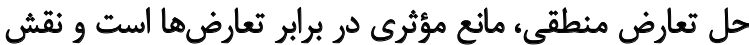

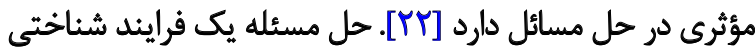

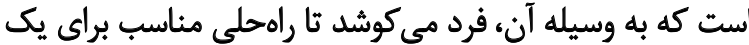

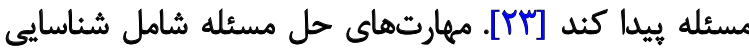

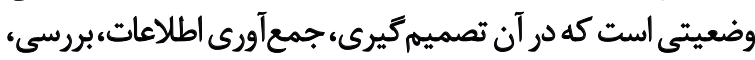

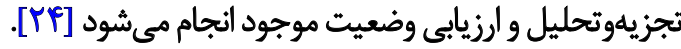
امروزه استفاده از مهارتهاي حل مسئله، مهارتى حياتى است و

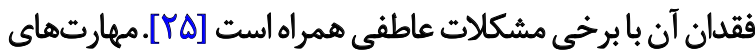

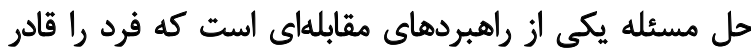

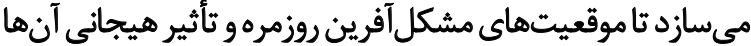

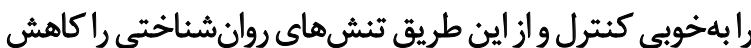

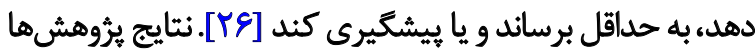


يرسش نامه استرس": اين مقياس راكوهن و همكاران در سال

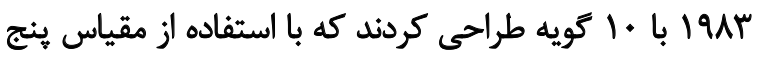

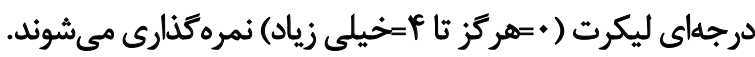

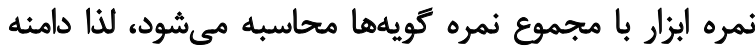

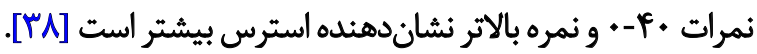

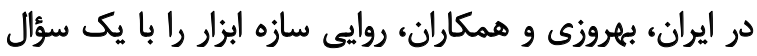

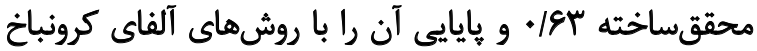

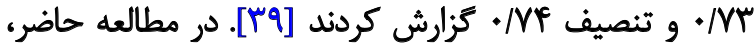

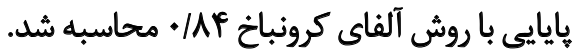

يرسشنامه مهارت حل مسئلهُ": اين مقياس را هينر و ويترسن

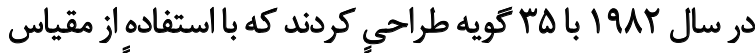

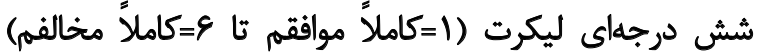

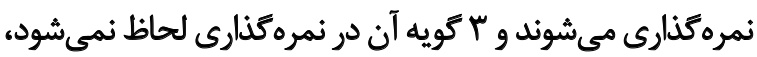

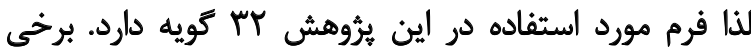

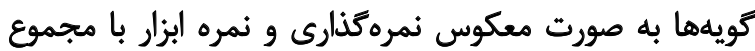

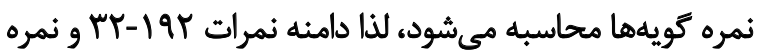

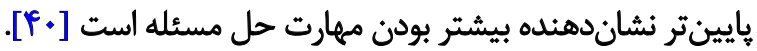

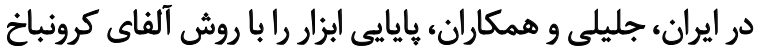

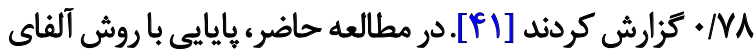

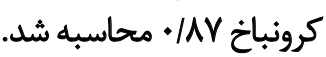

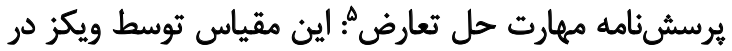

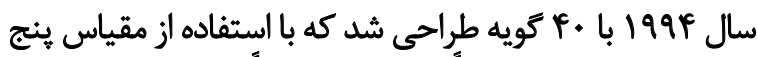

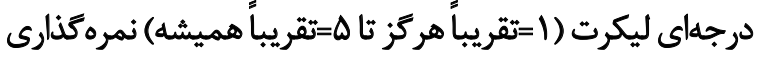

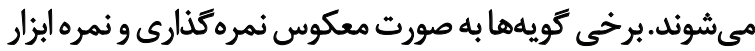

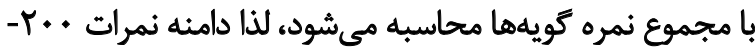

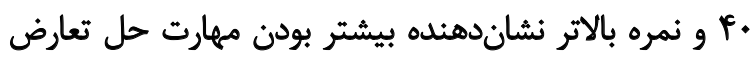

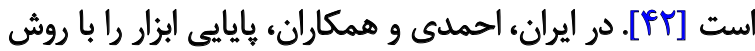

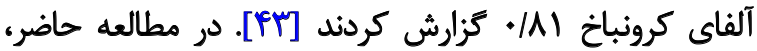

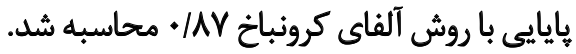

SPSS- دادهها در دو سطح توصيفى و استنباطي در نرمافزارهاي

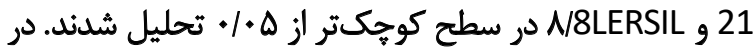

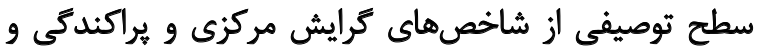

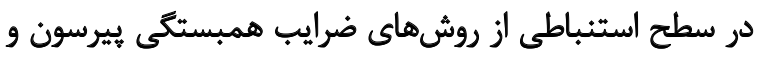
مدليابى معادلات ساختارى استفاده شد.

يافتهاها

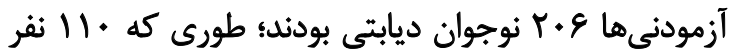

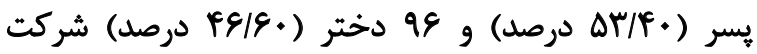

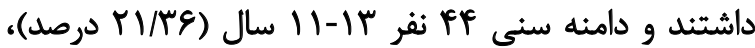

3. stress questionnaire

4. problem solving skills questionnaire

5. conflict resolution skills questionnaire
بيمارستان امام خمينى (ره) و مراجعلكنينده به يزشكان متخصص

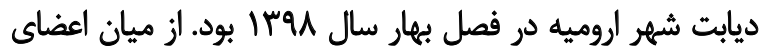

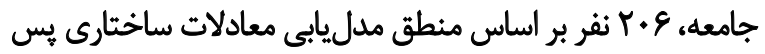

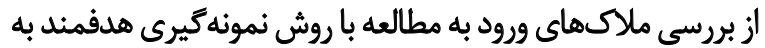

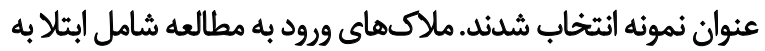

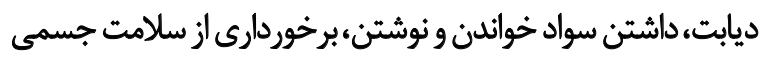

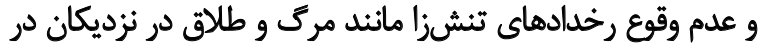

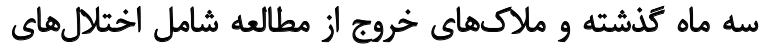

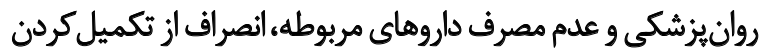

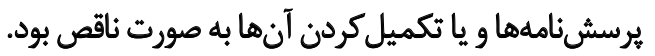
نحوه اجراى ثيروهش اينطور بود كه يس از تصويب برويوزال

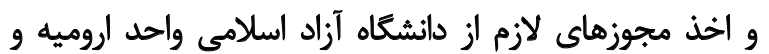

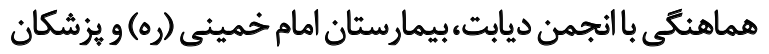

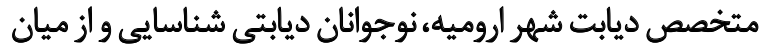

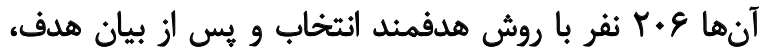

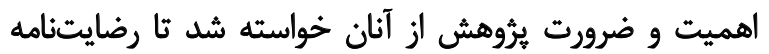

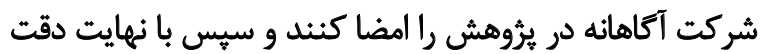

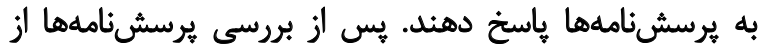

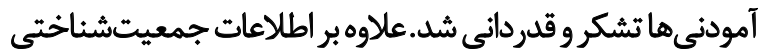

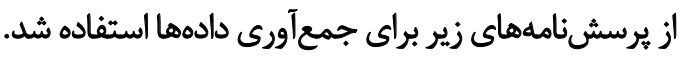
مقياس غربالكرى سوءمصرف مواد،نسخه نوجوانان ':اين مقياس

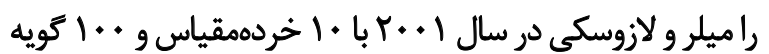

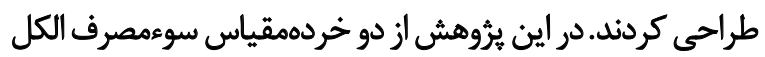

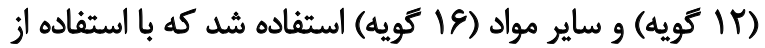

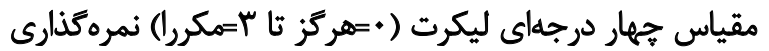

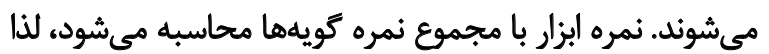

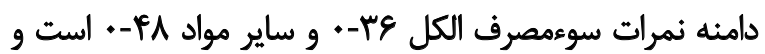

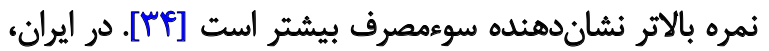

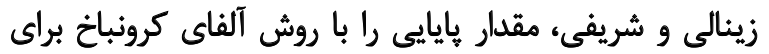

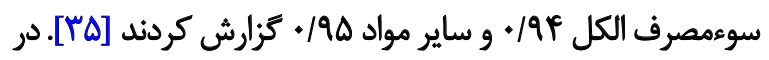

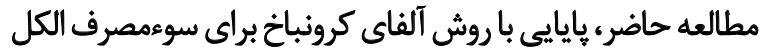

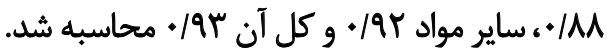

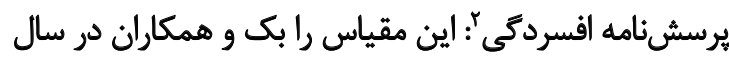

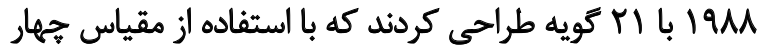

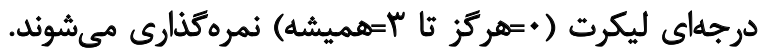

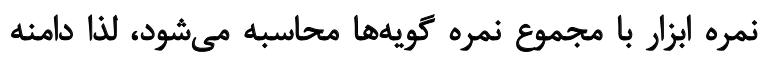

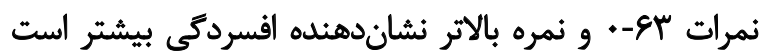

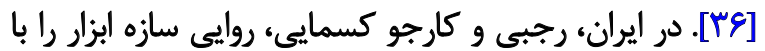

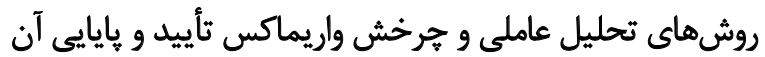

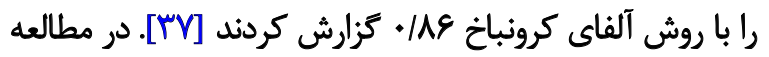

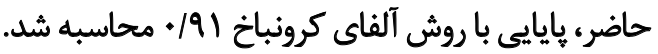

1. substance abuse screening scale-adolescents version

2. depression questionnaire 
تغييرات مهارت حل تعارض رادر نوجوانان ديابتى بيشبينى كنيند

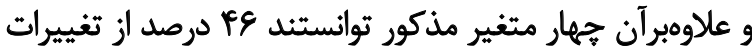

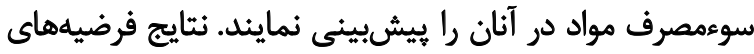

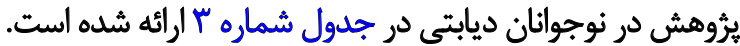

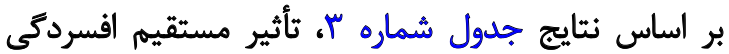

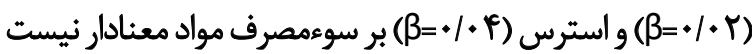

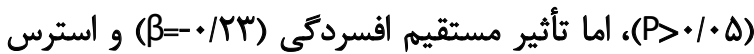

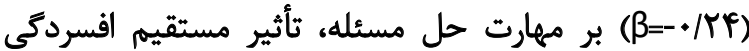

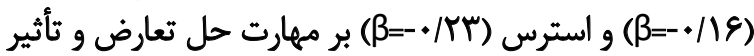

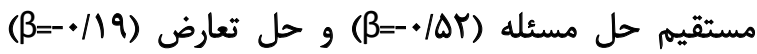

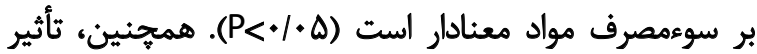

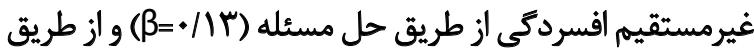

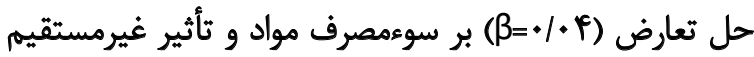

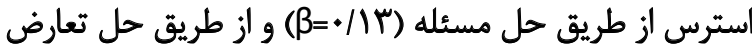

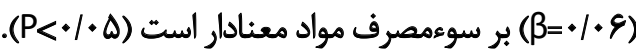

بs

با توجه به مشكلات روانشناختى فراوان در نوجوانان ديابتى

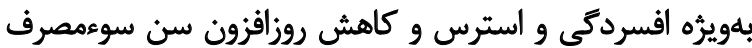

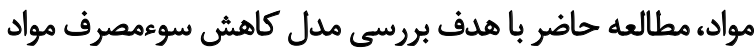

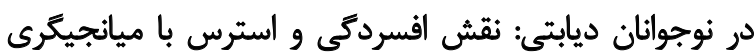

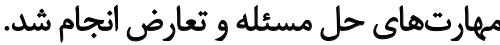

يافتهها نشان داد كه تأثير مستقيم افسردكى و استرس بر بران

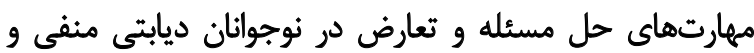

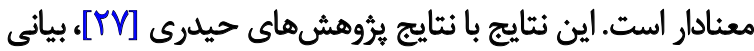

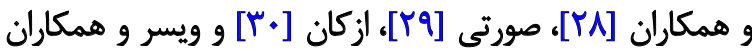

9 نفر 18-1f

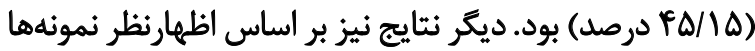

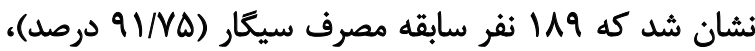

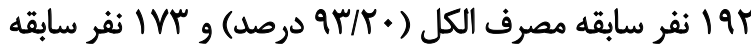

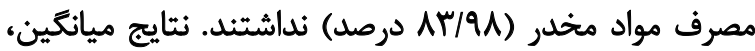

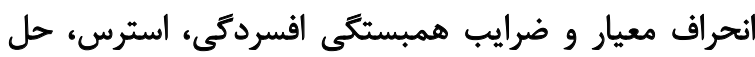

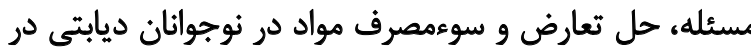
جدول شماره الرائه شده است. بر اساس نتايج جدول شماره ا، رابطه افسردكى و استرس

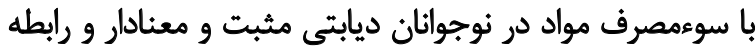

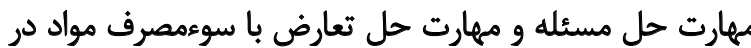

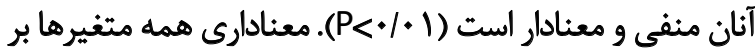

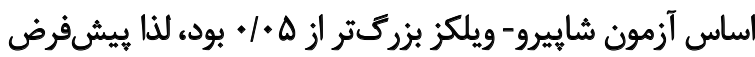

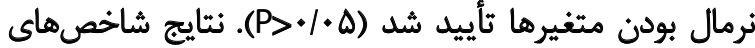

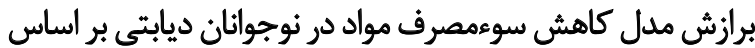

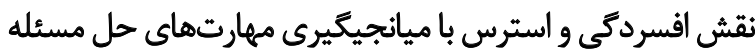
و تعارض در جدول شماره Y ارائه شده است.

بر اساس نتايج جدول شماره r، همه شاخصهاى برازش مدل

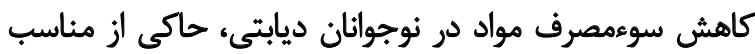

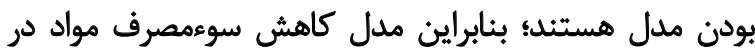

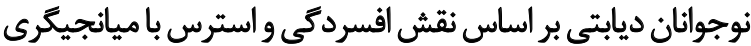

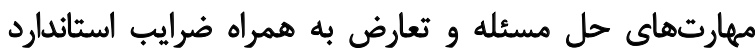

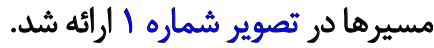

بر اساس نتايج تصوير شماره ا، متغيرهاى افسردكى و استرس إس

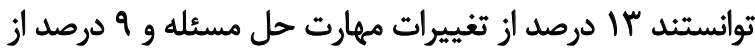

جدول ا. نتايج ميانكين، انحراف استاندارد و ضرايب همبستكى متغيرهاى يُروهش در نوجوانان ديابتى

\begin{tabular}{|c|c|c|c|c|c|c|}
\hline سوءمصرف مواد & حل تعارض & حل مسئله & استرس & أفسردكى & ميانكين+اندراف معيار & متغيرهاي برُوهش \\
\hline & & & & 1 & $r \varphi / \cdot+ \pm I \varphi / V A$ & 1. أفسردمى \\
\hline & & & 1 & $.115^{*}$ & $\mid V / F I \pm N+A$ & ك. السترس \\
\hline & & 1 &.$- / M r e *$ &.$- / T I^{* *}$ & IrQ/FYETYF/Eq & ب. حل مسئله \\
\hline & 1 & . & $-. / \pi+\infty$ & $-* / \pi I^{* *}$ & $\lambda) / F \Delta \pm \pi \Psi / / \gamma$ & F. جل تعارض \\
\hline 1 & $-+/ T \Lambda^{* *}$ & $-* / \Delta Y+*$ & $+/ \pi r^{* * *}$ & $\cdot /\left.M\right|^{* *}$ & 19/9 $\Delta \pm / 1 \%$ & هـ سوءمصرف مواد \\
\hline
\end{tabular}

$* * P<\cdot 1 \cdot 1, * P<\cdot 1 \cdot \Delta$

جدول Y. شاخص هاى برازش مدل كاهش سوءمصرف مواد در نوجوانان ديابتى

\begin{tabular}{|c|c|c|c|c|c|c|c|}
\hline RMSEA & NNFI & NFI & AGFI & GFI & CFI & $\chi 2 / d f$ & شاخصهاي برازش \\
\hline $.1 \circ V$ &.$/ 97$ & ./af & $\cdot / A V$ & $+/ 9 f$ & +/9V & $r / \cdot q$ & آماره \\
\hline $.1 \cdot \wedge<$ & $\cdot 19 \cdot>$ & $\cdot / 9 \cdot>$ & $\cdot \mid 1 \cdot>$ & $\cdot / 9 \cdot>$ & $\cdot / 9 \cdot>$ & $r<$ & حد قابل يذيرش \\
\hline
\end{tabular}


جدول ץ. نتايج فرضيههاي بثروهش در نوجوانان ديابتى

\begin{tabular}{|c|c|c|c|c|}
\hline 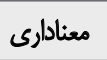 & 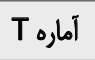 & خطاى استائدارد برآورد & ضريب مسير & فرضيهها \\
\hline $.1 . .1$ & $-M / M$ & $1+1$ & $-\cdot / \pi$ & افسردمى بر مهارت حل مسثله تأثير مستقيم دارد. \\
\hline $.1 .+1$ & $-M / P I$ & 1.9 &.$- / 4 f$ & استرس بر مهارت حل مسئه تأثير مستقيم دارد. \\
\hline.$/ 4$ & $-r / T r$ & $.1+\varphi$ &.$- / 18$ & افسردكى بر مهارت حل تعارض ثأثير مسثقيم دارد. \\
\hline $.1+1$ & - & 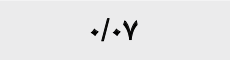 &.$- / 4 r$ & استرس بر مهارت حل تعارض تأثير مستقيم دارد. \\
\hline$\cdot M$ & $\cdot / \pi$ & $.1 . r$ &.$/ .4$ & افسردكى بر سوءمصرف مواد تأثير مستقيم دارد. \\
\hline - Ier & $+/ \uparrow 8$ & $.1+\Delta$ &.$/ \%$ & استرس بر سوءمصرف مواد تأثير مستقيم دارد. \\
\hline$+1+\infty$ & $-8 / M$ & $.1 \cdot 4$ & $-+/ \Delta r$ & مهارت حل مسئله بر سوءمصرف مواد تأثير مستقيم دارد. \\
\hline$+1+.4$ & $-r / Q$ &.$/ 6$ & $-+/ 19$ & مهارت حل تعارض بر سوءمصرف مواد تأثير مستقيم دارد. \\
\hline.$/ 1$ & $r / M F$ &.$/ \cdot v$ &.$/ \mathrm{r}$ & افسردكى از طريق مهارت حل مسئله بر سوءمصرف مواد تأثير غيرمستقيم دارد. \\
\hline.$/ . r$ & T/TE & $.1 \cdot r$ &.$/ \%$ & افسردكى از طريق مهارت حل تعارض بر سوعمصرف مواد تأثير غيرمستقيم دارد. \\
\hline.$/ . r$ & $t / N e$ & .1 .8 &.$/ r$ & استرس از طريق مهارت حل مسثله بر سوهمصرف مواد تأثير غيرمستقيم دارد. \\
\hline $.1 . . r$ & $r / \cdot 1$ & $\cdot 1 \cdot r$ & .1 .8 & استرس از طريق همارت حل تعارض بر سوهمصرف هواد تأثير غيرمستقيم دارد. \\
\hline
\end{tabular}

براي وقوع جالشها و عدم حل مناسب آنها هستند. نكته حائز

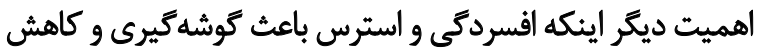

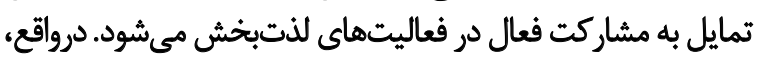

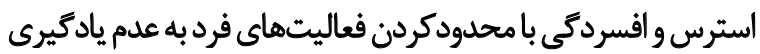

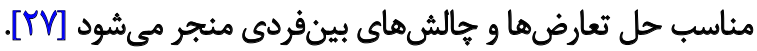

[اب[] همسو بود. در تبيين اين ثتايج مىتوان كفت كه افراد داراى

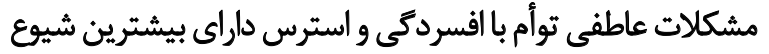

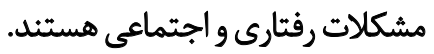
به عبارت ديكر، افراد مبتلا به افسردكى و استرس به دليل

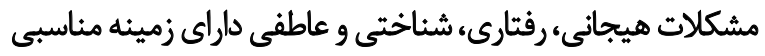

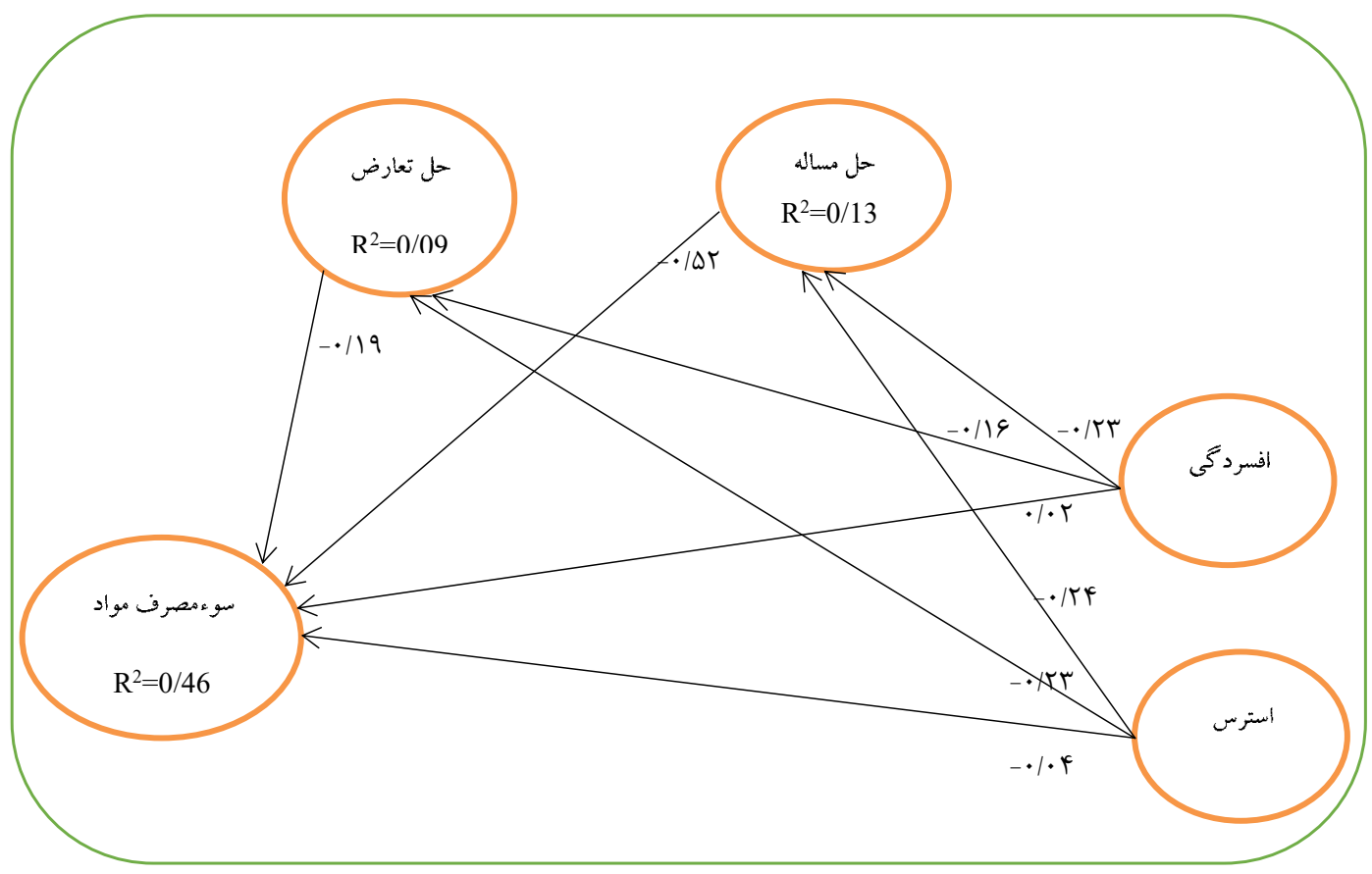

تصوير ا. مدل كاهش سوءمصرف مواد در نوجوانان ديابتى به همراه ضرايب استاندارد مسيرها 
انتظار داشت نوجوانانى كه از مهارتهاى حل مسئله و تعارض

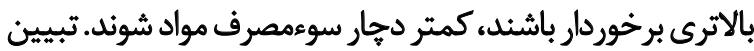

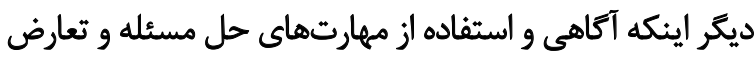

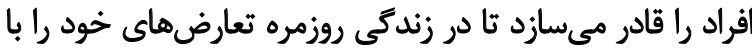

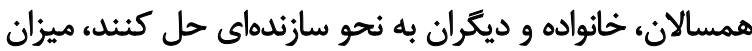

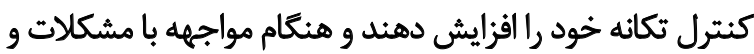

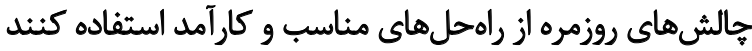

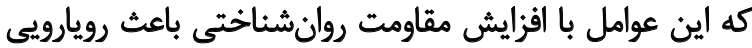

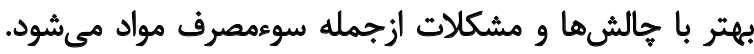

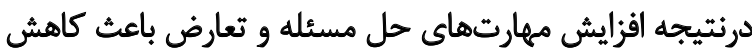
سوءمصرف مواد در نوجوانان مىشودي.

علاوهبراين، يافتهها نشان داد كه تأثير غيرمستقيم افسردكى و

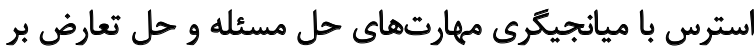

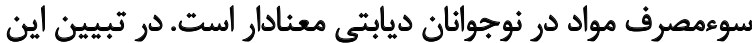

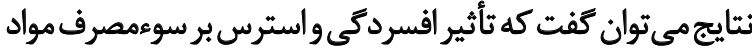

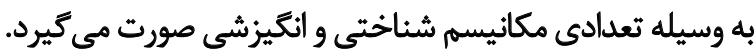

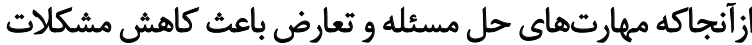

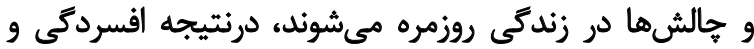

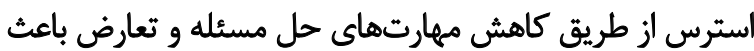

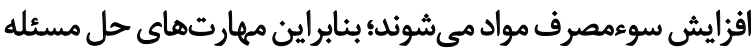

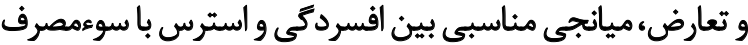
مواد در نوجوانان ديابتى است.

مههمترين محدوديتهاى يُروهش، محافظه كارى افراد در ارائه

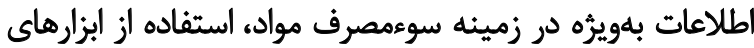

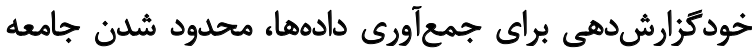

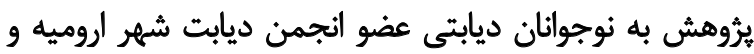

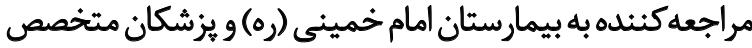

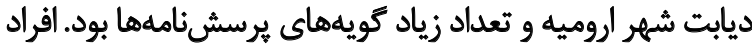

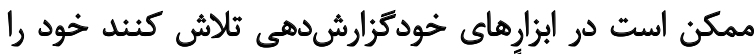

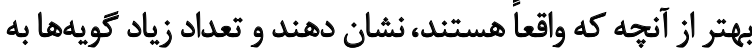

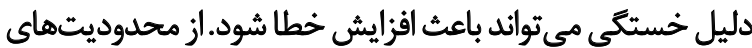

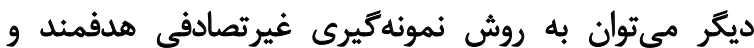

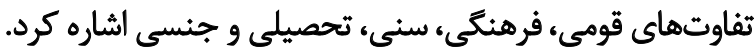

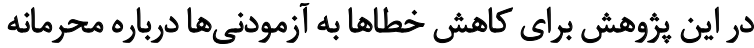

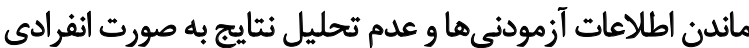

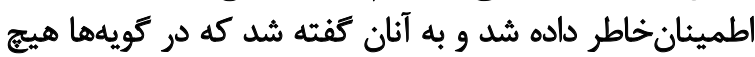

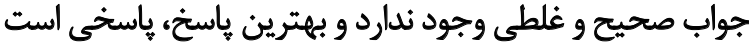
كه كوياى وضعيت واقعى آنان باشد.

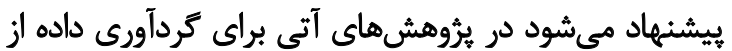

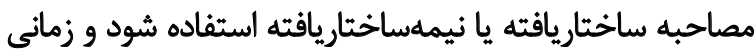

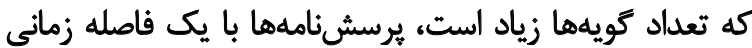

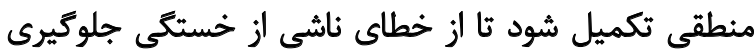

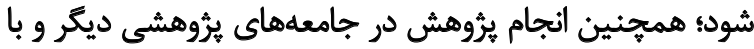

در اين بُروهش مشخص شد كه افسردگى و استرس بر مهارتهاي

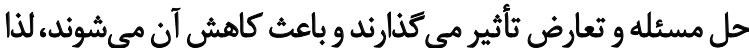

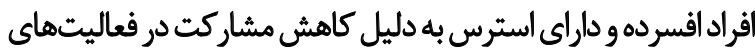

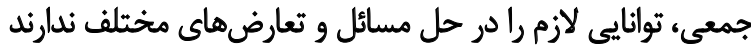

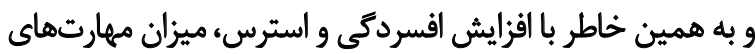
حل مسئله و تعارض در نوجوانان ديابتى كاهش مي ميابيد.

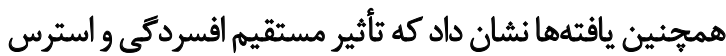

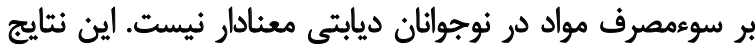

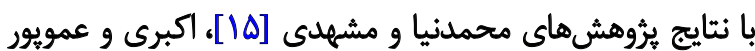

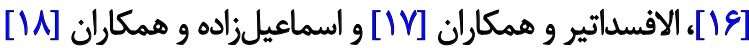

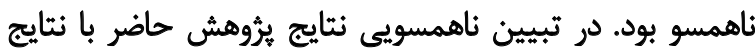

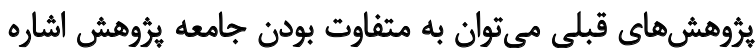

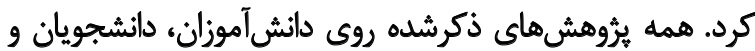

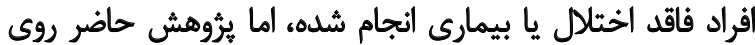

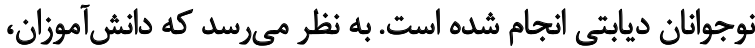

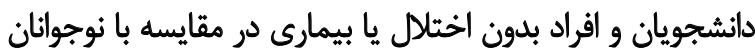

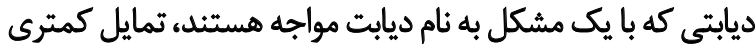

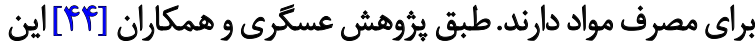

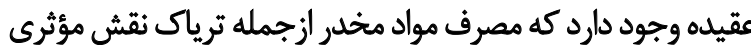

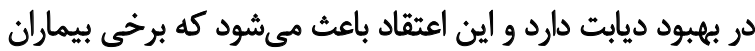

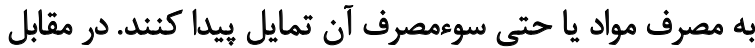

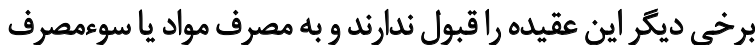

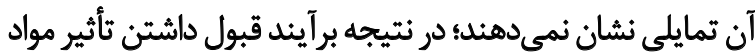

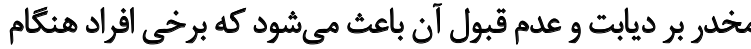

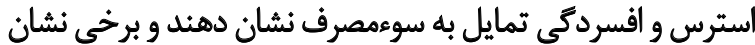

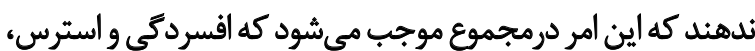

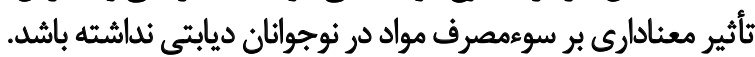
ديكر يافتهها نشان داد كه تأثير مستقيم مهارتهاى حل مل مسئله

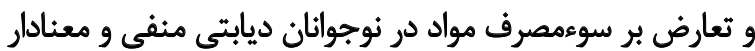

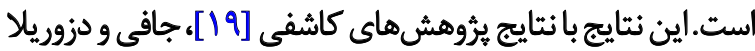

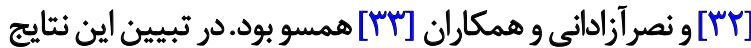

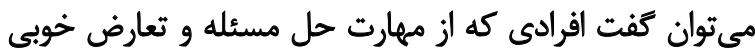

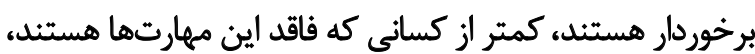

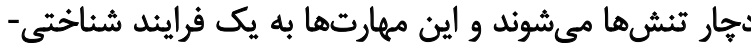

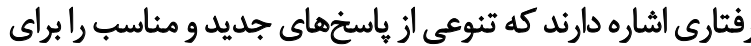

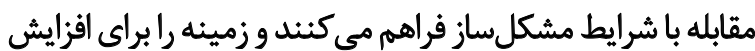

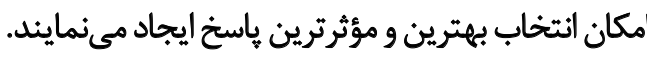

به عبارت ديكر، اين مهارتها يك فرايند درك و تفكر خلاق

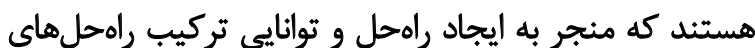

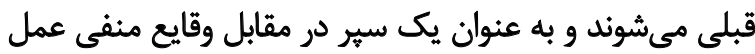

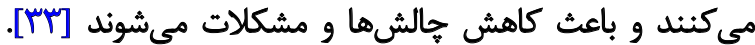

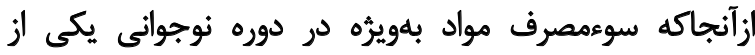

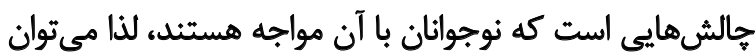




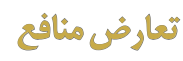

نويسندكان اعلام ميدارند كه هيجكونه تضاد منافعى در

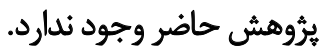

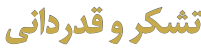

از رئيس و معاون بروهشى دانشعاه آزاد اسلامى واحد اروميه،

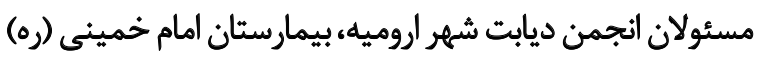

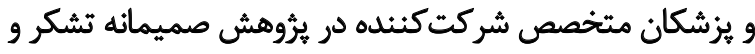

قدردانى مي شودن.
جامعهاي كستردهتر، امكان تصميم كيرى و برنامهريزى براي

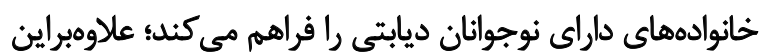

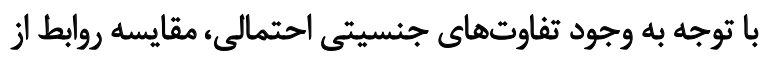

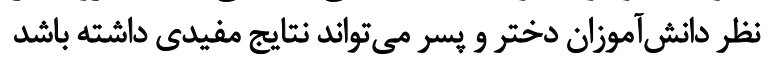

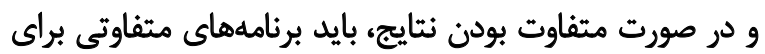

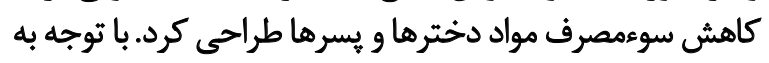

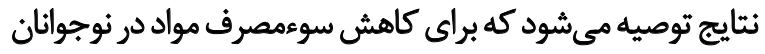

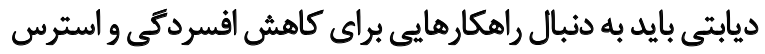

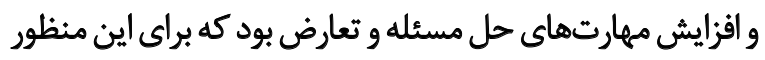

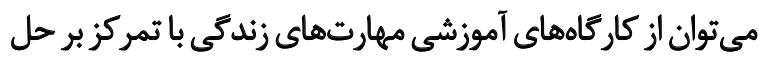

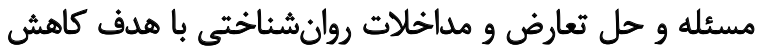
افسردگى و استرس استفاده كرد. نتيجليكيرى

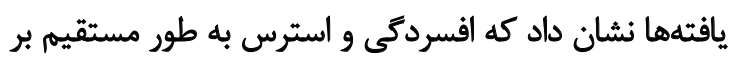

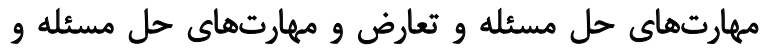

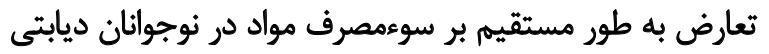

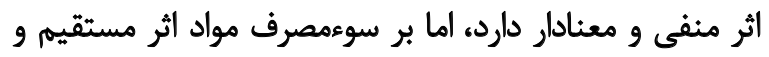

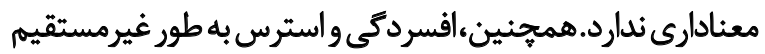

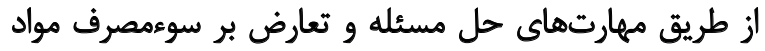

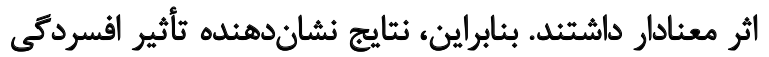

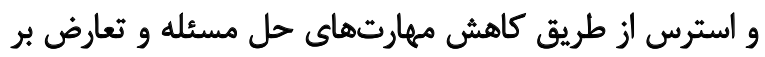
افزايش سوءمصرف مواد در نوجوانان ديابتى بود.

مالاحظات اخلاقى

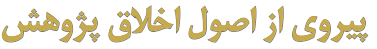

در اين يُروهش، تمامى اصول اخلاق در يُؤهش ازجمله اصل

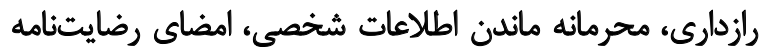

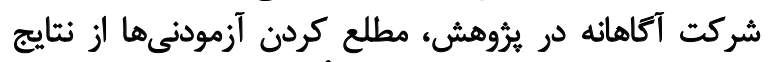

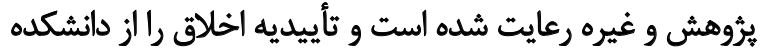

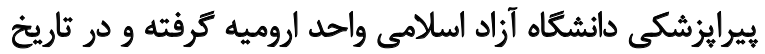

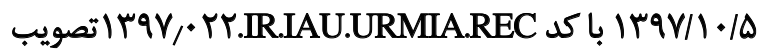

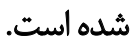
the

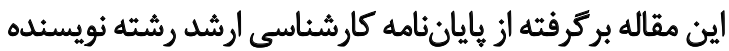

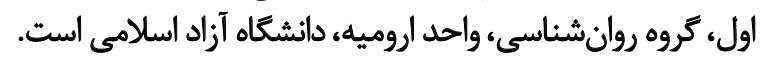

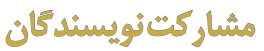

تمامي نويسندكان معيارهاى استاندارد نويسندكى بر اساس

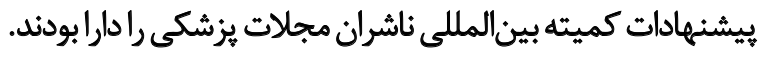




\section{References}

[1] Asadpour E, Veisi S. [The effectiveness of acceptance and commitment therapy on marital satisfaction, sexual self-esteem and burnout martial among women with type 2 diabetes (Persian)]. J Arak Uni Med Sci. 2019; 21(7):8-17. http://jams.arakmu.ac.ir/article-1-5710-en.html

[2] Walter-Holiner I, BArbarini DS, Lutschg J, Blassnig-Ezeh A, Zanier U, Saely $\mathrm{CH}$, et al. High prevalence and incidence of diabetic peripheral neuropathy in children and adolescents with type 1 diabetes mellitus: Results from a five-year prospective cohort study. Pediatr Neurol. 2018; 80:51-60. [DOI:10.1016/j.pediatrneurol.2017.11.017] [PMID]

[3] Ojo O, Wang X, Ojo OO, Ibe J. The effects of substance abuse on blood glucose parameters in patients with diabetes: A systematic review and meta-analysis. Int J Environ Res Public Health. 2018; 15(12):1-16. [DOI:10.3390/ijerph15122691] [PMID] [PMCID]

[4] Hampton WH, Hanik IM, Olson IR. Substance abuse and white matter: Findings, limitations, and future of diffusion tensor imaging research. Drug Alcohol. 2019; 197:288-98. [DOI:10.1016/j.drugalcdep.2019.02.005] [PMID] [PMCID]

[5] Jiang S, Wu L, Gao X. Beyond face-to-face individual counseling: A systematic review on alternative modes of motivational interviewing in substance abuse treatment and prevention. Addict Behav. 2017; 73:216-35. [DOI:10.1016/j.addbeh.2017.05.023] [PMID]

[6] Buttram ME, Kurtz SP, Ellis MS, Cicero TJ. Gabapentin prescribed during substance abuse treatment: The perspective of treatment providers. J Subst Abuse Treat. 2019; 105:1-4. [DOI:10.1016/j.jsat.2019.07.011] [PMID]

[7] Cornett EM, Budish R, Latimer D, Hart B, Urman RD, Kaye AD. Management of challenging pharmacologic issues in chronic pain and substance abuse disorders. Anesthesiol Clin. 2018; 36(4):615-26. [DOI:10.1016/j. anclin.2018.07.009] [PMID]

[8] Narimani M, Rajabi S. [The effect of EEG biofeedback on the reduction of depression, anxiety, stress and craving beliefs in individuals with substance abuse disorder (Persian)]. J Res Addict . 2012; 6(1):7-18. http:// etiadpajohi.ir/article-1-329-en.html

[9] Qi J, Yang X, Tan R, Wu X, Zhou X. Prevalence and predictors of posttraumatic stress disorder and depression among adolescents over 1 year after the Jiuzhaigou earthquake. J Affect Disord. 2019; 261:1-8. [DOI:10.1016/j.jad.2019.09.071] [PMID]

[10] Zahid JA, Grummedal O, Madsen MT, Gogenur I. Prevention of depression in patients with cancer: A systematic review and meta-analysis of randomized controlled trials. J Psychiatr Res. 2020; 120:113-23. [DOI:10.1016/j.jpsychires.2019.10.009] [PMID]

[11] Nathoo N, Mackie A. Treating depression in multiple sclerosis with antidepressants: A brief review of clinical trials and exploration of clinical symptoms to guide treatment decisions. Mult Scler Relat Disord. 2017; 18:177-180. [DOI:10.1016/j.msard.2017.10.004] [PMID]

[12] Wemm SE, Larkin C, Hermes G, Tennen H, Sinha R. A day-by-day prospective analysis of stress, craving and risk of next day alcohol intake during alcohol use disorder treatment. Drug Alcohol Depend. 2019; 204:1-7. [DOI:10.1016/j.drugalcdep.2019.107569] [PMID]

[13] Lgrip ML, Milivojevic V, Bertholomey ML, Torregrossa MM. Sexual dimorphism in the neural impact of stress and alcohol. Alcohol. 2018; 72:49-59. [DOI:10.1016/j.alcohol.2018.02.002] [PMID] [PMCID]

[14] Timmers I, Quaedflieg CWEM, Hsu C, Heathcote LC, Rovnaghi CR, Simons LE. The interaction between stress and chronic pain through the lens of threat learning. Neurosci Biobehav Rev. 2019; 107:641-55. [DOI:10.1016/j.neubiorev.2019.10.007] [PMID]
[15] Mohammad Nia S, Mashhadi A. [The effect of meaning of life on the relationship between attitude toward substance abuse and depression (Persian)]. Shefaye Khatam. 2018; 6(3):43-51. [DOI:10.29252/ shefa.6.3.43]

[16] Akbari B, Amoupour M. The relation depression with attitude drug abuse in high school students the Rasht city. J Educ Psychol. 2010; 1(2):720. https://www.sid.ir/en/journal/ViewPaper.aspx?id=265797

[17] Olafsdottir J, Hrafnsdottir S, Orjasniemi T. Depression, anxiety and stress from substance-use disorder among family members in Iceland. Nordic Studies on Alcohol and Drugs. 2018; 35(3):165-78. [DOI:10.1177/1455072518766129]

[18] Esmaeelzadeh S, Moraros J, Thorpe L, Bird Y. The association between depression, anxiety and substance use among Canadian post-secondary students. Neuropsuchiatr Dis Treat. 2018; 14:3241-51. [DOI:10.2147/ NDT.S187419] [PMID] [PMCID]

[19] Kashefi T. [The relationship between life skills and vulnerability to drug abuse among university students (Persian)]. J Torbat Heydariyeh Un Med Sci. 2013-4; 1(1):70-7. http://jms.thums.ac.ir/article-1-36-en.html

[20] Arrue M, Caballero S. Teaching skills to resolve conflicts with acute confusional syndrome patients in nursing using the Case Method (CM). Nurse Educ Today. 2015; 35(1):159-64. [DOI:10.1016/j. nedt.2014.09.006] [PMID]

[21] Fish JN, Maier CA, Priest JB. Substance abuse treatment response in a Latino sample: the influence of family conflict. J Subst Abuse Treat. 2015; 49:27-34. [DOI:10.1016/j.jsat.2014.07.011] [PMID]

[22] Wang M, Zhang D, Zhang L. Introduction of TRIZ theory for the conflict-solving in the building energy and environment management system innovation. Procedia Eng. 2015; 121:2232-9. [DOI:10.1016/j.proeng.2015.09.201]

[23] Huang J, Tan SP, Walsh SC, Spriggens LK, Neumann DL, Shum DHK, et al. Working memory dysfunctions predict social problem solving skills in schizophrenia. Psychiatry Res. 2014; 220(1-2):96-101. [DOI:10.1016/j. psychres.2014.07.043] [PMID]

[24] Pakarinen E, Kikas E. Child-centered and teacher-directed practices in relation to calculation and word problem solving skills. Learn Indiv Differ. 2019; 70:76-85. [DOI:10.1016/j.lindif.2019.01.008]

[25] Cevik YD. Assessor or assessee? Investigating the differential effects of online peer assessment roles in the development of students' problemsolving skills. Comput Human Behav. 2015; 52:250-8. [DOI:10.1016/j. chb.2015.05.056]

[26] Ertekin Pinar S, Yildirim G, Sayin N. Investigating the psychological resilience, self-confidence and problem-solving skills of midwife candidates. Nurse Educ Today. 2018; 64:144-9. [DOI:10.1016/j.nedt.2018.02.014] [PMID]

[27] Heydari S. [The prediction of students' depression disorder based on the process and content of family and problem solving skill (Persian)]. J Fam Health. 2015; 4(2):76-87. https://www.sid.ir/en/Journal/ViewPaper.aspx?ID=573134

[28] Bayani AA, Ranjbar M, Bayani A. [The study of relationship between social problem-solving and depression and social phobia among students (Persian)]. J Mazandaran Univ Med Sci. 2012; 22(94):91-8. https:// www.sid.ir/en/journal/ViewPaper.aspx?id=282015

[29] Sorati P. [The mediating role of perceived stress in the relationship between social problem solving styles and quality of work life among the teachers (Persian)]. J Res Behav Sci. 2018; 15(4):427-34. https://www. magiran.com/paper/1809270?lang=en 
[30] Ozkan Y. Anxiety, depression, problem solving and stress management in patients with ankylosing spondylitis. Meandros Med Dent J. 2018; 19:57-63. [DOI:10.4274/meandros.98704]

[31] Visser MM, Heijenbrok-Kal MH, Spijker AV, Oostra KM, Busschbach JJ, Ribbers GM. Coping, problem solving, depression, and healthrelated quality of life in patients receiving outpatient stroke rehabilitation. Arch Phys Med Rehabil. 2015; 96(8):1492-8. [DOI:10.1016/j. apmr.2015.04.007] [PMID]

[32] Jaffee WB, D'Zurilla TJ. Personality, problem solving, and adolescent substance use. Behav Ther. 2009; 40(1):93-101. [DOI:10.1016/j. beth.2008.03.001] [PMID]

[33] Nasrazadani E, Maghsoudi J, Mehrabi. The relationship of social problem-solving skills and dysfunctional attitudes with risk of drug abuse among dormitory students at Isfahan university of medical sciences. Iran J Nurs Midwifery Res. 2017; 22(4):276-279. [DOI:10.4103/ijnmr. IJNMR_58_16] [PMID] [PMCID]

[34] Miller FG, Lazowski LE. The adolescent SASSI-A2 manual: Identifying substance use disorders. Springville, IN: SASSI Institute; 2001.

[35] Zeinali A, Sharifi H. [Risk and protective factors in adolescent' alcoho and substance abuse (Persian)]. Urmia Univ Med Sci. 2018; 28(12):77082. http://umj.umsu.ac.ir/article-1-4214-en.html

[36] Beck AT, Steer RA, Garbin MG. Psychometric properties of the beck depression inventory: Twenty-five years of evaluation. Clin Psychol Rev. 1988; 8(1):77-100. [DOI:10.1016/0272-7358(88)90050-5]

[37] Rajabi Gh, Karju Kasmai S. [Psychometric properties of a Persian language version of the beck depression inventory second edition (Persian)]. Train Mea. 2013; 3(10):139-58. https://www.sid.ir/en/Journal/ ViewPaper.aspx?ID=336054

[38] Cohen S, Kamarck TA, Mermelstein R. A global measure of perceived stress. J Health Soc Behav. 1983; 24(4):385-96. [DOI:10.2307/2136404] [PMID]

[39] Behroozi N, Shahani Yeylaq M, Pourseyed SM. [Relationship between perfectionism, perceived stress and social support with academic burnout (Persian)]. Strategy Cult. 2013; 5(20):83-102. http://www.jsfc.ir/ article_43841_en.html

[40] Heppner PP, Petersen CH. The development and implications of a personal problem-solving inventory. J Counsel Psychol. 1982; 29(1):66-75. [DOI:10.1037/0022-0167.29.1.66]

[41] Jalili A, Hejazi M, Entesar Foumani G, Morovati Z. [The relationship between meta-cognition and academic performance with mediation role of problem solving (Persian)]. J Child Ment Health. 2018; 5(1):80-91. http://childmentalhealth.ir/article-1-379-en.htm

[42] Weeks D. The eight essential steps to conflict resolution. New York: Tarcger/Putman; 1994.

[43] Ahmadi SA, Safarzade H, Hoozoori M, Dehnavi F. [The role of cultura intelligence of managers on employees' conflict resolution ability (Persian)]. Soc Cogn. 2013; 2(3):101-16. [DOI:10.5267/j.msl.2013.06.037]

[44] Asgary S, Naderi Gh, Shahrezaei JR, Ahmadi P. [Comparison of plasma level of lipid peroxidation, lipids \& blood sugar in opium addicted \& control groups (Persian)]. J Qazvin Univ Med Sci. 2003; 26:115-19. http:// journal.qums.ac.ir/article-1-293-en.html 